\title{
Core-Collapse Supernova neutrino detection with KM3NeT
}

\author{
Marta Colomer Molla ${ }^{1,2, *}$ and Massimiliano Lincetto ${ }^{3, * *}$ \\ ${ }^{1}$ APC, Univ Paris Diderot, CNRS/IN2P3, CEA/Irfu, Obs de Paris, Sorbonne Paris Cité, France \\ ${ }^{2}$ IFIC - Instituto de Física Corpuscular (CSIC-Universitat de València), Paterna, Valencia, Spain \\ ${ }^{3}$ Aix Marseille Univ, CNRS/IN2P3, CPPM, Marseille, France
}

\begin{abstract}
Core Collapse Supernovae (CCSNe) are explosive phenomena that may occur at the end of the life of massive stars, releasing over $99 \%$ of the energy through neutrino emission. While the explosion mechanism is not fully understood, neutrinos are believed to play an important role. The only detection as of today are the 24 neutrinos from SN1987A. The observation of the next Galactic CCSN will lead to important breakthroughs in astroparticle physics.

For a Galactic CCSN, the KM3NeT ORCA and ARCA detectors in the Mediterranean Sea will observe a significant neutrino signal via the detection of Cherenkov light, mostly induced by Inverse Beta Decay interactions in sea water. The detection of coincident photons by the 31 photomultipliers of each $\mathrm{KM} 3 \mathrm{NeT}$ digital optical module (DOM) allows for an efficient discrimination of the optical backgrounds.

The KM3NeT detection sensitivity to a Galactic CCSN and the potential to resolve the neutrino light-curve have been estimated relying on detailed Monte Carlo simulations. Specific criteria are proposed for the online triggering and the participation in the SNEWS network.
\end{abstract}

\section{Introduction}

The KM3NeT collaboration is currently building two Cherenkov detectors in the Mediterranean Sea, ORCA and ARCA, each composed of Digital Optical Modules (DOMs) housing 31 3-inch photomultipliers (PMTs) [1]. Groups of 18 DOMs are arranged into flexible vertical detection lines and an array of 115 lines constitutes a building block. ORCA, off the French coast, will consist of one block with about $9 \mathrm{~m}$ separation between DOMs and and about $20 \mathrm{~m}$ separation between lines while ARCA, offshore Sicily, will consist of two blocks with $36 \mathrm{~m}$ vertical and $90 \mathrm{~m}$ horizontal spacings, correspondingly [2].

Although KM3NeT detectors are mainly designed for high-energy neutrino detection, the $10 \mathrm{MeV}$-scale neutrino signal from a CCSN can be identified as a simultaneous increase of the counting rate in the photomultipliers of the detector. The main interaction modes of these $\mathrm{MeV}$ neutrinos in water are Inverse Beta Decay, Elastic Scattering and interactions of neutrinos with oxygen atoms. The outgoing electron/positron produced in these interactions induces the production of Cherenkov light that can be detected by the PMTs.

\footnotetext{
*e-mail: mcolomer@apc.in2p3.fr

**e-mail: lincetto@cppm.in2p3.fr
} 


\section{Flux models and simulations}

A state-of-the-art 3D simulation of the accretion phase of a $27 \mathrm{M}_{\odot}$ and an $11 \mathrm{M}_{\odot} \mathrm{CCSN}$ have been considered in this work [3,4]. This simulation provides the time dependent CCSN neutrino spectrum, which is a quasi-thermal distribution which depends on the average neutrino energy $\tilde{E}_{v}$, the neutrino luminosity $L(t)_{S N}^{v}$ and the spectral pinching shape parameter, $\alpha$. The output of this 3D simulation has been used to compute the CCSN neutrino interaction rate by multiplying the flux with the latest cross section measurements provided in $[5,6]$.

A full MC simulation of CCSN neutrinos in KM3NeT has been produced and used to estimate the expected detection rate in the detector for a CCSN at an arbitrary distance, considering background rates as observed with the first ORCA and ARCA detection lines.

${ }^{40} \mathrm{~K}$ decays in sea water are one of the main background sources and they can be distinguished from a CCSN signal using coincidences between the different PMTs of a given DOM. The number of PMTs in a DOM detecting a photon within a time window of $10 \mathrm{~ns}$ is called multiplicity (M) and is used to reject the ${ }^{40} \mathrm{~K}$ background, which dominates at low multiplicities. Dedicated simulations have shown that selecting events in the multiplicity range 6 $\leq \mathrm{M} \leq 10$ optimizes the signal-to-background ratio.

KM3NeT preliminary

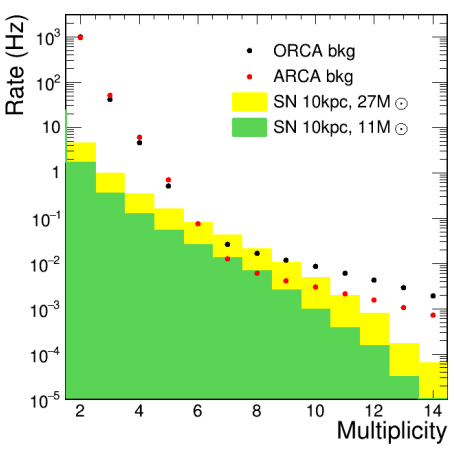

KM3NeT preliminary

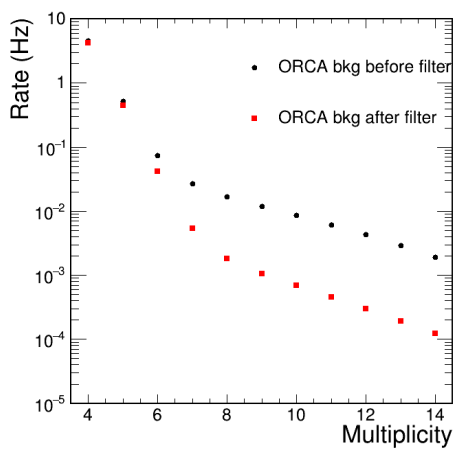

Figure 1. On the left, DOM background rates as a function of the multiplicity for ORCA (black squares) and ARCA (red squares) from data and expectations for CCSN signal at $10 \mathrm{kpc}$ for the $27 \mathrm{M}_{\odot}$ (yellow filled) and the $11 \mathrm{M}_{\odot}$ (green filled) progenitors. On the right, ORCA rates before (black dots) and after applying the muon filter (red squares).

Atmospheric muons are another prominent background producing Cherenkov tracks over long distances with high multiplicity events on multiple DOMs. Suppression of $M \geq 4$ correlated coincidences between two or more DOMs on a few $\mu$ s time scale is efficient to reject the muon background. Based on this, a muon filter has been developed and optimized for ORCA.

On Fig. 1 (left) shows the multiplicity rates per DOM for signal simulations and background for both KM3NeT detectors, ARCA and ORCA. The signal rates are computed from the total number of detected events during the $550 \mathrm{~ms}$ simulation duration. The effect of the muon filter on the ORCA background rates above $\mathrm{M}=4$ is shown on the right plot.

\section{Significance results}

The number of signal and background events after applying the multiplicity cut and the muon filter are used to compute the significance as a function of the distance to the source. 
The results for ORCA, ARCA and the combination of both detectors are shown in Fig. 2 for the two stellar progenitors considered in this study. Both the statistical uncertainties and the more relevant systematic errors, originating from the uncertainties on the models (flavor conversion [4], different simulation codes [7], neutrino opacities [7], observer direction [9]), have been taken into account. A combined significance of $5 \sigma$ is achieved at $25 \mathrm{kpc}$ for the 27 $\mathrm{M}_{\odot}$ progenitor, ensuring the coverage of the full Galaxy. In the case of the $11 \mathrm{M}_{\odot}$ progenitor, a significance of $5 \sigma$ is reached beyond the Galactic Centre with the full ORCA detector.

KM3NeT preliminary

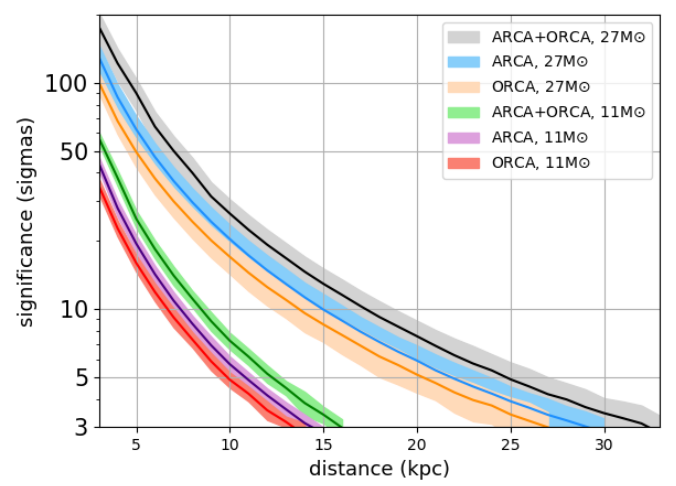

Figure 2. Detection significance of a CCSN neutrino signal as a function of the distance to the source.

\section{Trigger performance}

An approach for the real-time trigger of a CCSN neutrino burst with KM3NeT has been evaluated and will be operational with the next phases of the detector deployment.

Data from the deployed ARCA and ORCA lines have been extensively analyzed by sampling the background on a $\tau=100 \mathrm{~ms}$ time scale in order to identify patterns in the DOM response that could mimic the CCSN signal.

A robust trigger is implemented by defining the trigger level as the number of DOMs that detect a multiple coincidence in the multiplicity cut over a larger sliding time window of $n$ times $\tau$, with $n$ an integer allowing to cover the signal duration.

The triggering capability of the approach for the ORCA detector is summarised in Fig. 3 as a function of the CCSN distance for the two progenitors studied. The $\leq 1 /(2$ weeks $)$ fake trigger requirement to participate in the SNEWS global alert network [8] is highlighted in the same plot. A CCSN of $27 \mathrm{M}_{\odot}$ could be triggered with full ORCA up to $18.5 \mathrm{kpc}$ and above the Galactic Centre for the $11 \mathrm{M}_{\odot}$ CCSN.

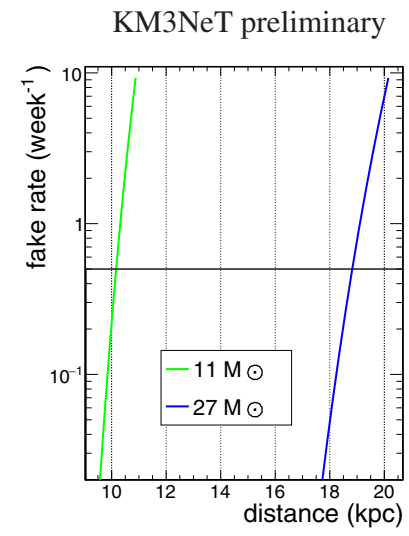

Figure 3. Estimated false trigger rates in ORCA for a background level equal or greater to the expectation value of signal plus background as a function of the CCSN distance for the two progenitors studied. The black line indicates the SNEWS limit. 


\section{Sensitivity to fast time variations on the neutrino light-curve.}

Latest 3D simulations predict anisotropic hydrodynamical instabilities during the CCSN accretion phase, which may play an important role in the efficient neutrino heating [9] [10].

The focus has been placed here on the Standing Accretion Shock Instability (SASI) that could enhance the energetic final explosion. This model leaves as a footprint fast time variations in the neutrino light-curve around $200 \mathrm{~ms}$ after the core bounce, with a characteristic oscillation frequency [4].

The detected CCSN neutrino light-curve has been simulated for the $27 \mathrm{M}_{\odot}$ progenitor at 5 $\mathrm{kpc}$, for an observation on the privileged direction of the SASI sloshing. All hits are included. Background has been included assuming a Poisson distribution, which is a preliminary an quite optimistic assumption. As in [11], a Fourier analysis has been used to recover the SASI frequency and the significance to the SASI peak has been estimated through MC pseudoexperiments. A $3 \sigma$ sensitivity to the oscillation peak is obtained with one KM3NeT building block at $5 \mathrm{kpc}$ for the $27 \mathrm{M}_{\odot}$ progenitor. A more detailed description of the background will be implemented in a subsequent step.

\section{Conclusions}

The response of the KM3NeT neutrino detectors to core-collapse supernova neutrinos has been estimated by means of a complete Monte Carlo simulation and an exhaustive study of the background from the first KM3NeT datasets.

The KM3NeT combined sensitivity for a future CCSN detection has been estimated to be of $5 \sigma$ at $25 \mathrm{kpc}$ (coverage of the full Galaxy) for a $27 \mathrm{M}_{\odot}$ stellar progenitor. Assuming an $11 \mathrm{M}_{\odot}$ stellar progenitor, a significance of $5 \sigma$ is reached beyond the Galactic Centre with a single building block.

As for the online triggering capabilities, the maximum triggering distance meeting the SNEWS false alert rate requirement has been estimated to be well beyond the Galactic Centre for the two progenitors studied.

\section{Acknowledgements}

This project is partly supported by the LabEx UnivEarthS exploratory project E9: Low energy astrophysics with KM3NeT.

\section{References}

[1] KM3NeT Collaboration, Eur. Phys. J. C (2016) 76:54 (2015)

[2] KM3NeT Collaboration, Journal of Physics G 43 (8), 084001 (2016)

[3] I. Tamborra et al., Phys. Rev. D 90, 045032 (arXiv e-prints 1406.0006) (2014)

[4] I. Tamborra et al., Phys. Rev. Lett. 111,121104 (arXiv e-prints 1307.7936) (2013)

[5] A. Strumia and F. Vissani, Phys. Lett. B 564 (2003) 42

[6] G. Radel and R. Beyer, Mod. Phys. Lett. A, 08 (1993) 12

[7] , K.Kotake and T.Takiwaki The Astrophysical Journal, 853(2) 170

[8] K.Scholberg, "The Supernova Early Warning System", (arXiv:0803.0531) (2008)

[9] A. Marek, H.-T. Janka and E. Müller, Astron. Astrophys. 496, 66 (2009)

[10] H.T.Janka, "Neutrino-driven Explosions", arXiv:1702.08825 (2017)

[11] J.Miganda, "Detecting Fast Time Variations in the Supernova Neutrino Flux with HyperKamiokande", arXiv:1609.04286 (2015) 